\title{
The discrimination of vibratory patterns on a tactile matrix
}

\author{
ELLEN F. GOTTHEIL, ROGER W. CHOLEWIAK, and CARL E. SHERRICK \\ Princeton University, Princeton, New Jersey 08540
}

\begin{abstract}
The ability of observers to discriminate static vibrotactile patterns was examined on a tactile matrix of 64 vibrators. Observers were presented with pairs of patterns that varied in symmetry, number of pattern elements (numerosity), and number of common pattern elements (communality), and were asked to judge whether the members of the pair were the same or different. Errors were primarily related to communality, while variation in symmetry had no effect on performance. Only at high levels of communality did numerosity affect performance differentially. Confusions resulted when few of many elements in the patterns differed. The levels of performance were found to be inferior to those reported by investigators who examined such relationships in patterns presented to more widespread body loci. A discussion of models of confusion in such tasks is presented.
\end{abstract}

In their attempts to devise auditory or visual prosthetic devices, investigators have often attempted to construct systems in which the tactile sense may be used as a replacement or supplement. One approach has been simply to present the skin with mechanical vibrations correlated with certain dimensions of acoustic signals (Aston \& Weed, 1971; Gault, 1930; Pickett \& Pickett, 1963) or of optical signals (Bliss, Katcher, Rogers, \& Shepard, 1970; Collins, 1970). In order to investigate the factors contributing to both the successes and limitations of such systems, it is important to determine how patterns are discriminated from each other on a tactile matrix of the type empolyed by Bliss et al.(1970) and by Collins (1970) in their sensory substitution systems.

The ability of the skin to discriminate static vibratory patterns (i.e. patterns that vary among themselves only spatially rather than spatiotemporally) was studied by Geldard and Sherrick (1965). In their experiment, 10 vibrators were placed at widely dispersed positions on the observer's body to maximize the discriminability of individual vibrators. Observers discriminated 500 "same" and 500 "different" pairs of patterns, in which the number and location of the active elements was varied. Analysis of the data was based on two factors: numerosity and communality. The term "communality" refers to the identity of elements between successive patterns to be compared; the greater the communality, the greater the similarity, and the more obscure the change between patterns. The data revealed that increased communality and numerosity of pattern elements resulted in a greater number of errors in the discrimination of patterns.

Repetition of the Geldard and Sherrick (1965) study

This study was conducted under Grant NS-04755 from the National Institutes of Health, DHEW, to Princeton University. Requests for reprints should be sent to Roger W. Cholewiak, Department of Psychology, Princeton University, Princeton, New Jersey 08540. by Gilson (1968) on the fingers confirmed the original findings. In a similar experiment involving patterns generated by a tactile Vocoder, Aston and Weed (1971) also obtained the general communality-error relation.

It is reasonable to ask whether communality would be important in a situation in which the patterns to be discriminated did not consist of carefully isolated stimulus points, but of closely spaced points within a tactile matrix.

In addition to communality and numerosity, there is a third feature of spatial patterns that should be investigated on a tactile matrix. In visual pattern discrimination, a structure that is symmetrical about an axis is generally judged to be a "better" figure than one composed of random elements (Attneave, 1955; Handel \& Garner, 1966). Slight alterations from symmetry are easily discriminated. This may be a general feature of pattern perception and may obtain for the skin as well as for the eye. Thus, pattern symmetry might be expected to affect tactile pattern discrimination.

With the aid of computer-generated patterns presented to the fingers, Hill (Note 1) explored the effect of organization on observers' ability to recall the location of the stimulated elements. Analyzing responses as a function of spatial organization of pattern elements (presumably ordered according to standards of preferred visual organization), Hill reported that such organization did not importantly affect the accuracy of performance. However, studies done on the body or fingers with widely spaced stimulators are open to Kirman's (1973) criticism: "One may imagine what sort of limited visual organization would be possible with 10 retinal loci randomly distributed or arranged in a row. Such a display would hardly reveal the extraordinary capacities of the visual system for form perception" (p. 64).

Employing a tactile matrix one may investigate the way in which patterns are discriminated in systems such as Bach-y-Rita's tactile television substitution system (TVSS) (Collins, 1970) or Bliss' optical-to-tactile converter (Optacon) (Bliss et al., 1970), in which informa- 
tion is presented in pictorial fashion to a large number of closely packed vibrators in contact with the skin. When no effort is made to minimize spatial interaction, does the cutaneous system process stimulus elements in the same way that it seems to process a smaller number of widely scattered vibrators on the body or on separate fingers? If it does, it would be expected that discrimination errors would be correlated with increases in communality between patterns and with increases in numerosity. It is possible that when spatial interaction within a field takes place, the skin will process spatial stimuli in much the same manner as the visual system. If so, it might be predicted that discriminations including symmetrical spatial stimuli should be facilitated. Since there have been no previous studies exploring the nature of such discriminations on a tactile matrix, many patterns should be tested in combination so that the effects of factors associated with densely packed arrays might be investigated.

\section{METHOD}

\section{Apparatus}

A PDP-8/f computer controlled the production of patterns on a 64-Bimorph bender vibratory matrix. Contactors were on $15-\mathrm{mm}$ centers in the 8 by 8 vibrator array. The matrix was brought into contact with the ventral thigh of a seated observer, and the contactor surface shaped and clamped to the body contour. After the matrix had been fitted, a 640-g weight was placed on the matrix to provide about $10 \mathrm{~g}$ of force per contactor.

The vibrotactile patterns were presented for $250 \mathrm{msec}$ by specifying a repetition of $10250-\mathrm{Hz}$ haversine pulses at intervals of $25 \mathrm{msec}$ on each of the pattern elements. The activation time of $250 \mathrm{msec}$ was chosen in order to have a clear signal and yet to minimize adaptation. A 1-sec interstimulus interval was chosen to be sufficiently long to avoid the complicating effects of temporal summation or apparent motion (Kirman, 1974) and short enough not to strain tactile short-term memory (Hill \& Bliss, 1968). After the two patterns had been presented, the observer indicated whether the two were the same pattern, or different patterns by depressing designated keys on a numeric keypad.

\section{Observers}

The 10 observers in the experiment included eight undergraduates at Princeton University who served as paid volunteers and two members of the professional research staff.

\section{Stimuli}

Numerosity. Sixteen patterns were constructed at two levels of numerosity, resulting in a total set size of 32 patterns. Previous studies of acuity on this matrix indicated that variations of one contactor might not be perceived (Geldard \& Sherrick, Note 2), so patterns were built from blocks of 2 by 2 adjacent contactors, transforming the matrix into a 4 by 4 block matrix. The two levels of numerosity consisted of four-block (16-contactor) patterns and eight-block (32-contactor) patterns. The two levels of numerosity were subjectively matched for loudness by the method of adjustment. The loudness matching guarded against confusion of the effects of numerosity with the effects of loudness or of spread of excitation.

In order to maintain a relatively constant level of pattern density, the matrix was divided into quadrants. In the four-block condition, there was one activated block per quadrant, and in the eight-block condition, there were two activated blocks per quadrant.

Symmetry. At each level of numerosity, both symmetrical and random patterns were desired. Symmetrical patterns were generated by reflecting the patterns of either one block (fourblock numerosity) or two blocks (eight-block numerosity) of the upper left-hand quadrant in both the vertical and horizontal axes. Random patterns had the same density per quadrant as the symmetrical patterns, but the positions of the blocks in each quadrant were randomly determined. Illustrative examples of the patterns are shown in Figure 1. Three levels of symmetry interaction were tested: random vs. random, random vs. symmetrical, and symmetrical vs. symmetrical.

Communality. Communality is defined as the ratio, expressed as percent, of shared elements to total elements in a particular pattern (see Figure 1). All 16 patterns at each level of numerosity were paired with each other, so that each pattern was equally represented in the sample. The total stimulus set consisted of 480 pairs, half of which were "sames," half "differents," of varying levels of communality. The communalities of the "differents" at the four-block level were $0 \%, 25 \%, 50 \%$, and $75 \%$, and, at the eight-block level, $0 \%, 25 \%, 37.5 \%, 50 \%$, $62.5 \%, 75 \%$, and $87.5 \%$.

The 480 pairs were randomly ordered and presented to the observer in eight sessions of 60 trials each, 4 trials at each level of numerosity. Upon responding "same" or "different," the observer was presented with the next pattern pair after a 1-sec postresponse delay. The presentation of numerosity and communality conditions was randomized and counterbalanced across observers to control for possible order effects.

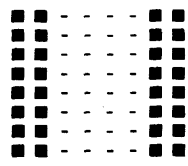

A

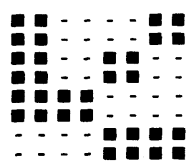

c
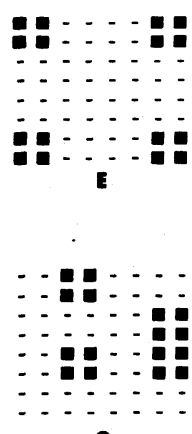

G
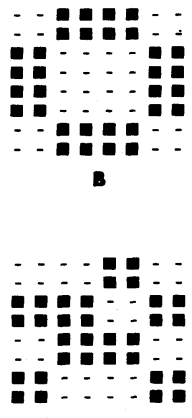

D
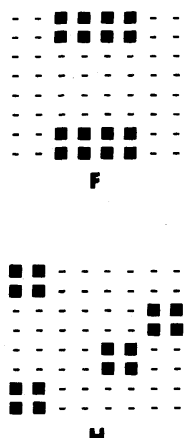

Figure 1. Sample of the static patterns employed in the study of pattern discrimination and communality. Patterns A-D are eight-block patterns, while E-H are four-block patterns. Patterns A, B, E, and F are symmetrical and the remaining are random. Representative communalities for patterns pairs are: $A$ and $B=50 \% ; A$ and $C=62.5 \% ; C$ and $D=37.5 \% ; F \& G=25 \%$. 


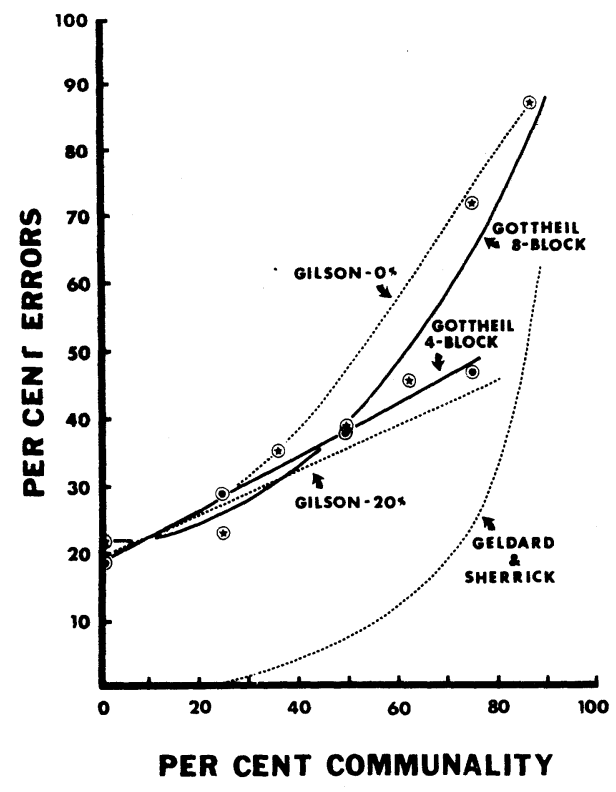

Figure 2. Plot of average percent error for two numerosities (four-block and eight-block) as a function of percent communality. Functions from Geldard and Sherrick (1965) and Gilson (1968) are also shown for comparison.

\section{Results}

A plot of raw error rates may be observed at each level of numerosity in Figure 2. Raw error rates were plotted in order to facilitate comparison with the functions of Geldard and Sherrick (1965) and Gilson (1968), who plotted raw error scores.

Fitting a line by the method of least squares to the corrected error scores for the four-block patterns yielded a linear relationship with the equation, $y=.16 x+18.4$ where $y$ is the percent error and $x$ is the percent communality. The data from the eight-block patterns were found by the least-squares method to fit an exponential equation better than a linear equation: $y=19.14 \exp (.01 x)$. For the four-block patterns, $r^{2}=.96$, and for the eightblock patterns, $r^{2}=.91$, indicating that most of the variance can be explained by the communality factor.

Figure 2 indicates that there was not a simple relationship between the four- and eight-block discriminations. If there had been an overall deterioration in performance on the eight-block patterns, two functions would be expected that were similar in appearance and separated by some constant amount due to a constant difference in error rates. Such was clearly not the case, for at low levels of communality, performance was roughly similar for the two numerosities. The Wilcoxon signed-rank test was used to compare the scores of the 10 subjects on four- and eight-block patterns, and at $0 \%, 25 \%$, and $50 \%$ communality the differences were not found to be significant. At $75 \%$ communality, however, the difference in performance on the fourand eight-block patterns was found to be significant $(\mathrm{p}<.02)$.
An analysis of variance was computed to analyze further the relationship between numerosity, communality, and error rate. In order to normalize the data, arc-sine transformations were taken of the error rate at each level of numerosity and communality interaction $(0 \%, 25 \%, 50 \%$, and $75 \%)$. The communalityerror rate main effect was found to be highly significant $(p<.001)$. The numerosity error rate main effect was not found to be significant $(p=.292)$. There was a significant Communality by Numerosity interaction $(p=.013)$. Analysis of the effect of symmetry yielded no significant results.

The graph obtained by Geldard and Sherrick (1965) plotted in Figure 2 is clearly exponential. Aston and Weed (1971) reported an equation similar to that of Geldard and Sherrick, although with different yintercepts (baseline error rates). Figure 2 also shows a comparison of the functions obtained in this study for the four- and eight-block patterns to the functions obtained in Gilson's (1968) study, analyzed according to "percent shift" of elements across hands. In these cases, it can be seen that data comparable to those of the present study were obtained.

\section{DISCUSSION}

It is clear that communality as defined here is the primary basis for correct discrimination of spatial patterns. The relationship between communality and error rate was, however, affected by the total number of pattern elements activated. The curves for the two levels of numerosity, relating percent communality to error rate, are not simple functions of each other. At the four-block level, error rate increased linearly with communality, whereas at the eight-block level, error rate and communality were found to have an exponential relationship.

Gilson (1968) analyzed the discrimination errors made in his study in terms of percent shift of pattern elements across hands. Error rate decreased as a greater percentage of elements moved across hands between patterns in a pair. It is evident that the function obtained for shifts between eight-block patterns in the present study corresponds in shape, slope, and baseline error rate to the exponential function obtained by Gilson for his $0 \%$ shift condition, in which no elements changed hands. The function obtained for shifts between four-block patterns resembles in shape, slope, and baseline error rate the linear function obtained in Gilson's $20 \%$ and $30 \%$ shift conditions.

In our 16-block matrix, four-block patterns activated $25 \%$ of the matrix, while eight-block patterns activated $50 \%$ of the matrix. Unshared elements in the four-block patterns had more available locations to which they could move between patterns than did unshared elements in the eight-block patterns. In other words, unshared elements might be "more different" in the four-block patterns than in the eight-block patterns. Gilson's (1968) finding that error rate decreased as a greater number of pattern elements shifted hands could also be interpreted as resulting from the unshared pattern elements being "more different" when they shifted hands. It appears important, therefore, to take into account not only pattern communality, but also the extent of the difference between unshared pattern elements when evaluating the ease or difficulty of a particular discrimination between two patterns.

The results of the experiments of Alexander (1963), who studied patterns presented to five electrodes on the lower arm, suggest how the difference between unshared patterns might be perceived. Each pattern consisted of from one to four simul- 
taneous electrical pulses. Alexander found that most subjects differentiated patterns on the basis of changes in the center of gravity between patterns. As the number of elements in the patterns increased, the ability to discriminate patterns was reduced.

The results of Alexander's (1963) study have obvious implications for both Gilson's (1968) and the present study. A larger percent shift between hands or from one area of the matrix to another could result in a greater shift in the center of gravity between patterns. Indeed, decreased communality could be interpreted as an increased potential for a shift in the center of gravity between patterns. At high levels of communality and numerosity, however, shifts in the center of gravity might become very difficult to perceive.

Hill (Note 1) described a "spreading model" to explain the errors made in reporting the location of stimulus elements in patterns presented to the fingers. Most of the errors were found to result from confusions between neighboring locations; that is, between adjacent fingers of the same hand or adjacent positions within a finger. With increasing numerosity, subjects reported fewer positions at the correct location and reported more in the surrounding area. Hill's "spreading model" may help to explain the increase in error rate at high levels of numerosity observed in all the cited studies of tactile pattern discrimination on a limited number of vibrators. At high levels of numerosity, it is more likely that stimulation will spread to neighboring locations and obscure the change between two patterns. It is possible that only at high-communaltiy levels, when differences between patterns are more subtle, do the effects of spreading become important.

At around $60 \%$ communality for our eight-block curve, and also for the Aston and Weed (1971), Geldard and Sherrick (1965), and Gilson (1968) curves, error rate begins to increase sharply. It is possible that the exponential function represents the interaction of two processes around this $60 \%$ point. One function might correspond to the almost linear, gradual increase in error rates at lower levels of communality and which, for low levels of numerosity, is constant throughout the spectrum of communality. Discriminations could be made on the basis of shift in the center of gravity, or the extent to which unshared elements are truly "different." A second process leading to rapid deterioration in discrimination may be indicated by the much steeper slope at high levels of communality for the higher numerosities. At this point, perhaps as a result of Hill's (Note 1) "spreading," patterns cease to be perceived as different.

The present results encourage us to respond to Kirman's (1973) inquiry into the potential revelations possible with dense arrays of stimulators as opposed to those distributed across the body. His argument that organization in the array should lead to a greater degree of discriminative capability appears not to apply when the comparison between Geldard and Sherrick's (1965) function in Figure 2 is made with those of the present study. Indeed, for the stimuli employed in these studies, the distributed body array allows for much better discriminative capability than does the compact tactile matrix. The introduction of temporal variation into the pattern display is currently being investigated as a method of improving the discrimination of vibratory patterns on the tactile matrix (Sherrick \& Cholewiak, Note 3).

\section{REFERENCE NOTES}

1. Hill, J. W. The perception of multiple tactile stimuli (Tech. Rep. 4823-1). Stanford, Calif: 1967.

2. Geldard, F. A., \& Sherrick, C. E. Princeton Cutaneous Project Progress Report No. 27. Princeton, N.J: March 1976.

3. Sherrick, C. E., \& Cholewiak, R. W. Matching speech to vision and touch. Paper presented at the conference on Speech-Analyzing Aids for the Deaf. Washington, D.C: Gallaudet College, 1977.

\section{REFERENCES}

Alexander, L. B. Spatial pattern perception and electrical excitation of the skin. Master's thesis. Pittsburgh, Pa: Carnegie Institute of Technology, 1963.

Aston, R., \& WEeD, H. A man-machine model for evaluation of the tactile Vocoder. Journal of the Association for the Advancement of Medical Instrumentation, 1971, 5, 19-25.

Attneave, F. Symmetry, information, and memory for patterns. American Journal of Psychology, 1955, 68, 209-222.

Bliss, J. C., Katcher, M., Rogers, C., \& Shepard, R. Optical-to-tactile image conversion for the blind. IEEE Transactions on Man-Machine Systems, 1970, MMS-11, 58-65.

Collins, C. C. Tactile television: Mechanical and electrical image projection. IEEE Transactions on Man-Machine Systems, 1970, MMS-11, 65-71.

GAULT, R. H. On the effect of simultaneous tactual-visual stimulation in relation to the interpretation of speech. Journal of Abnormal and Social Psychology, 1930, 24. 498-517.

Geldard, F. A., \& Sherrick, C. E. Multiple cutaneous stimulation: The discrimination of vibratory patterns. Journal of the Acoustical Society of America, 1965, 37, 797-801.

Gilson, R. D. Some factors affecting the spatial discriminations of vibrotactile patterns. Perception \& Psychophysics, 1968, 3, 131-136.

HANDEL, S., \& GARNER, W. R. The structure of visual pattern associates and pattern goodness. Perception \& Psychophysics, 1966, 1, 33-38.

Hill, J. W., \& Bliss, J. C. Modeling a tactile sensory register. Perception \& Psychophysics, 1968, 4, 91-101.

Kirman, J. H. Tactile communication of speech: A review and analysis. Psychological Bulletin, 1973, 80, 54-74.

Kirman, J. H. Tactile apparent movement: The effects of interstimulus onset interval and stimulus duration. Perception \& Psychophysics, 1974, 15, 1-6.

Picketr, J., \& Picketr, B. Communication of speech sounds by a tactile Vocoder. Journal of Speech and Hearing Research, 1963, 6, 207-210.

(Received for publication September 19, 1977.) 